\title{
ENFERMERÍA Y GLOBALIZACIÓN
}

\section{NURSING IN THE ERA OF GLOBALIZATION}

\author{
Dra. Tatiana Paravic KLIJN *
}

\begin{abstract}
RESUMEN
El siglo XX se caracterizó por una serie de cambios en diversos ámbitos de la vida humana y marcó el auge y desarrollo que tuvo Enfermería en el mundo. Es en este período donde surgieron corrientes de pensamiento que postularon la globalización, como un intento de respuesta social y económica al problema mundial de la pobreza. Es un fenómeno que va adquiriendo trascendencia en las diversas disciplinas. El progreso tecnológico y la mayor velocidad son parte de este proceso, y se hace cada vez más necesario un paradigma de acción, donde nuestra mayor preocupación sea el propio hombre. Hoy día, la preparación de la enfermera en este siglo XXI presenta grandes desafíos de competencias cognitivas y de habilidades prácticas, y responsabilidad por el cuidado de salud del otro, pero lo más necesario e importante es la mirada humanista y ética que guíen el comportamiento en esta aldea global.
\end{abstract}

Palabras clave: Globalización, enfermería, humanismo.

\begin{abstract}
The XX century was characterized by a series of changes in diverse areas of human life and it marked the rise and development that nursing has reached in the world. During this period, certain lines of thought emerged that posited globalisation as a social an economic response to the world problem of poverty. It is a phenomenon that has transcended different discipline. Technological progress, and the increased speed are part of this process and it is becoming more necessary an action oriented paradigm where our principal concern is the human being. Today, the nurse education for the XXI century presents great challenges related to cognitive skills, practical abilities and responsibility for the care of others. However, the most important and urgent needs are humanistic and ethical aspects to guide behaviour in this global village.
\end{abstract}

Key words: Globalization, nursing, humanism.

Fecha recepción: 07/10/09 Fecha aceptación: 14/12/09

\section{INTRODUCCIÓN}

El siglo XX, el de los grandes cambios, se caracterizó por la obtención de una mayor libertad civil, justicia y conciencia social e importantes descubrimientos en diferentes campos, tales como el espacio, la genéti- ca, la energía nuclear, la clonación de seres entre otros. Las estructuras sociales, políticas y económicas de los países, así como los avances en la ciencia médica, los progresos tecnológicos en los hospitales y la creciente demanda de los cuidados de salud, la Primera y la Segunda Guerra Mundial, provocaron 
numerosos cambios en la formación y en las actividades de las enfermeras (1). Las guerras despertaron la conciencia de la dependencia que tenían los países involucrados en éstas, respecto de sus enfermeras, ya que muchas vidas dependían de ellas. Los cuidados de enfermería marcaron la diferencia en la recuperación de los soldados enfermos y heridos. Comienza la proliferación de los hospitales con la función de tener que cubrir las demandas de la población enferma, es así que el cuidado se orienta hacia la enfermedad.

En los primeros 50 años de ese siglo, la prioridad estaba en erradicar las enfermedades transmisibles y controlar las infecciones por la utilización de métodos de asepsia y antisepsia. La concepción de salud estaba dada por la ausencia de la enfermedad y el cuidado estaba dado más bien por el curar centrado en algún órgano, y derivada de la práctica médica (2). Entre los años '50 y '60 aparece con mayor fuerza la enfermera sanitaria o comunitaria, cuyo énfasis era los cuidados domiciliarios y la educación sanitaria para desarrollar las potencialidades, proporcionar seguridad y alivio a las enfermedades de la población.

Se abrieron múltiples escuelas de enfermería en distintos lugares del mundo, y se dieron los pasos necesarios para organizar el conocimiento de esta profesión (1). Las fuerzas internas de la enfermería permitieron aumentar la conciencia y la participación para orientarse hacia la profesionalización y a la innovación del ámbito de sus responsabilidades.

Los movimientos científicos, tecnológicos y sociales del siglo XX han tenido un efecto significativo sobre el desarrollo de los cuidados de salud y sobre las políticas de los servicios de enfermería. Estos le han permitido al hombre prolongar y mejorar su calidad de vida.

Este entorno cambiante creó la necesidad de una formación rigurosa con el fin de mantenerse al día con el mundo moderno. Se exige la graduación de la enseñanza me- dia como requisito de ingreso a estudiar Enfermería, y en muchos países del mundo se debe rendir además la prueba para el ingreso a las universidades, donde es impartida esta carrera profesional. Aparecen los programas de especialización en Enfermería en distintos campos, y se asienta en la estructura de la formación de la enfermera el grado de licenciado (1), lo que le ha permitido acceder a los grados de magíster y doctor en su propia disciplina así como en otras. Comienza un impulso muy grande hacia la investigación con los programas de postgrado, las enfermeras comienzan a producir sus propios textos y revistas, aparecen las teorías de enfermería y se discute profusamente la ciencia de la enfermería.

El haber llegado hasta acá no ha estado exento de conflictos. El desarrollo de la Enfermería ha significado una permanente lucha global por permitirnos ir avanzando en el conocimiento y su aplicación, a pesar de tener un efecto significativo sobre la calidad de vida de la comunidad.

\section{DESARROLLO}

Es durante este siglo XX donde comienzan a surgir corrientes de pensamiento que postulan la conveniencia de crear la aldea global, como un mecanismo de mejor aprovechamiento de los recursos disponibles, pero que en la práctica no ha estado exenta de problemas.

La globalización surge en un contexto de políticas crecientes de apertura en el comercio exterior y en las relaciones económicas internacionales, en un contexto de desarrollo de las telecomunicaciones, fenómeno que evoluciona a una velocidad acelerada: tales como el fax, internet, telefonía, computación, entre otros, y en el contexto del desarrollo de la industria del transporte, tanto aéreo, marítimo como terrestre.

Las nuevas tecnologías y la eliminación 
de barreras nos permiten un nivel de acercamiento que no había existido antes, y por lo tanto un mayor acceso a la cultura y a los productos de otros países. Nos conocemos mejor y ello es positivo para evitar prejuicios y conflictos, lo que facilitaría la paz y el progreso. Entonces es nuestra responsabilidad construir una sociedad con mejor calidad de vida y mas segura para todos. El premio Nóbel de Economía de 1998 (3), decía: "La globalización ha enriquecido al mundo en un sentido científico y cultural", lo que sin duda hace más difícil, pero a su vez más desafiante la tarea de generar saberes sólidos y provechosos.

La globalización es un fenómeno trascendente que cada vez adquiere mayor importancia, tanto en la Economía como en la Sociología y en otras disciplinas de las Ciencias Sociales y de Salud.

La globalización surge como un intento de respuesta social y económica al problema mundial de la pobreza, recursos escasos y necesidades múltiples (4).

Esta globalización acelerada de hoy se caracteriza, primero, por la rapidez del progreso científico, su difusión y aplicación a los sectores más diversos debido a la revolución de la información. Se dice que en el 2020 el conocimiento global se multiplicará cada 6 meses, lo que significa que se sabrá el doble de lo que se sabía hace 180 días. Segundo, por las restricciones cada vez más severas impuestas a la migración de las personas. Es ésta una de las mayores carencias en la respuesta colectiva a los desafíos de la globalización. Este problema exige responder con humanidad, es decir con solidaridad y plena conciencia, otorgando dignidad al hombre y sentido a nuestra sociedad $(5,6)$.

La globalización avanza no siempre con coherencia, lo que ha estancado la progresión de la unidad del mundo y de la felicidad del hombre. Hay que canalizarla hacia el logro de una mayor justicia, solidaridad y unidad en el mundo. Hay que promover el crecimiento y el desarrollo humanos en sus políticas económicas para mejorar la distribución de los ingresos y las desigualdades.

Un escritor contemporáneo nos dice: "Nadie refutará que la globalización plantea muchos retos de índole política, jurídica, administrativa, sin duda. Y ella, si no viene acompañada de la globalización y profundización de la democracia, la legalidad y la libertad, puede traer también serios perjuicios, facilitando, por ejemplo, la internacionalización del terrorismo y de los sindicatos del crimen. Pero comparados a los beneficios y oportunidades que ella trae, sobre todo para las sociedades pobres $\mathrm{y}$ atrasadas que requieren quemar etapas a fin de alcanzar niveles de vida dignos para los pueblos, aquellos retos, en vez de desalentarnos, deberían animarnos a enfrentarlos con entusiasmo e imaginación. Y con el convencimiento de que nunca antes, en la larga historia de la civilización humana, hemos tenido tanto recursos intelectuales, científicos y económicos como ahora para luchar contra los males atávicos: el hambre, la guerra, los prejuicios y la opresión"(7).

La globalización nos exige conocer los valores, creencias, costumbres, necesidades, gustos, idiomas de los diferentes pueblos, por lo que se hace indispensable tener talentos humanos altamente calificados y preparados en las diversas áreas de la vida. Con capacidades creativas y de innovación, flexibles pero también rigurosos (8). Las universidades deben abrirse a este tipo de formación superior brindando opciones que generen y aumenten los conocimientos superiores así como la comprensión de estos fenómenos con el fin de contribuir a su propio bienestar y el de su comunidad (9).

La inversión continua en el capital humano, es un elemento clave para promover el desarrollo económico, la cohesión social y la equidad en el conocimiento.

Desde el prisma de la Enfermería el desarrollo de los talentos en esta disciplina requiere además de un compromiso a la calidad de vida de las personas bajo un paradigma que 
es por esencia humanista.

El humanismo en términos generales se define como un actitud centrada en el interés de lo humano, en el pleno desarrollo y bienestar de lo que el hombre es, desde las miradas biológicas, psicológica, social, cultural y espiritual.

Practicar el humanismo es conocerse uno mismo: es el autoconcepto reflejado en otra persona. El humanismo implica el respeto a la libertad interna del propio hombre para pensar sentir y creer.

El humanismo se establece en la reflexión: la preocupación máxima del hombre debe ser el propio hombre y todo lo que significan sus intereses sus problemas y su posición en la vida, constituyéndose en el ideal por desarrollar en cada individuo lo que hay específicamente de humano.

En este paradigma, el ser humano fundamentalmente es bueno, es libre de elegir y es responsable de sí mismo. Cada persona es única y capaz de maximizar su potencial y debe ser vista en forma holística (10). La fe en nuestras potencialidades y en la de los demás permitirá nuestro propio desarrollo y crecimiento que incidirá en aquéllos que necesiten de nuestros cuidados.

Enfermería ha heredado de su proceso histórico una filosofía de responsabilidad para el cuidado de la vida humana derivada del humanismo que le da el sustento y el significado de su práctica, para lograr un nivel satisfactorio de desarrollo y de salud en el ser humano (11).

El cuidado de enfermería es el cuidado de la salud del otro. Se sitúa en medio de la interacción de costumbres, creencias, valores y actitudes heredados del pasado, de una historia personal y particular, rodeados de símbolos. Este cuidado se hace posible cuando confluyen y se encuentran las personas que participan en la relación. Los valores están incorporados a la vida misma, conformando entre otros el concepto de cultura (12).

El cuidado de enfermería debe también ser productivo, lo que significa humano y de ca- lidad; lo que implica utilizar el conocimiento científico, y analizar con enfoque holístico de modo que la situación pueda articularse plenamente al contexto social, económico y legal, para tomar la mejor decisión sobre la actuación que se ofrecerá al cliente, paciente o usuario (13).

Colliere (14) expresa que cuidar o preocuparse por alguien, creer en alguien, reforzar sus capacidades, permitirle recobrar la esperanza, acompañarle en su experiencia de salud-enfermedad estando presente, son acciones invisibles. Pero éstas requieren de muchas competencias (15) ya que cuidar requiere de acciones pensadas y reflexionadas, requieren de recursos intelectuales para tomar decisiones y de ciertas habilidades específicas.

El cuidado y la preocupación implica también responsabilidad, que significa dispuesto a responder y debe ser guiado por el conocimiento, y este conocimiento debe estar motivado por la preocupación de ver a la otra persona en sus propios términos (10).

El cuidado profesional que significa diagnosticar y tratar los problemas de salud requiere de conocimientos, actitudes y destrezas que sólo se adquieren con estudios sistemáticos y ordenados, además de profundos conocimientos del hombre, de la salud, de la sociedad y de enfermería.

La calidad en la formación del profesional depende no sólo de los conocimientos y habilidades, sino también de los intereses y valores que regulan su actuación profesional. El amor a la profesión, la responsabilidad, el humanismo y la honestidad constituyen valores esenciales, reguladoras de la actuación de un profesional competente, que se reflejan en cada persona de manera diferente en función de su historia individual, sus intereses y sus capacidades (16).

Consubstantivo al hombre, tenemos la conciencia ética. Es imposible pensar en un ser humano carente de ella, como sería igualmente imposible pensarlo sin capacidad de raciocinar, de reflexionar, de retener 
en la memoria de un modo vivo el pasado, de proyectar el futuro, de indagar el sentido de la vida (17). Nadie puede negar que experimenta cotidianamente una existencia ética o moral, como un conjunto de ideas y criterios de acuerdo a los cuales conduce su vida, sus decisiones y comportamientos, la que es posible denominar como ética natural.

La ética se preocupa de los actos humanos en cuanto ellos conducen a la realización del bien en la persona, es por ello una efectiva orientación práctica para todos los trabajadores en salud, y en especial para las enfermeras, especialmente en este mundo de hoy, de grandes avances científicos y tecnológicos, donde el tiempo adquiere una importancia capital, pero donde aún temblamos ante el dolor, nos emocionamos ante el nacimiento de un niño y respetamos el paso de la vida a la muerte.

Especialmente hoy en día, Enfermería debe tener particular interés en la formación ética de sus alumnos, futuros profesionales, ya que continuamente serán sometidos a situaciones que reclamen de ellos una postura y opción ética, que exige algo más que el ejercicio de una ética espontánea o innata o una ética especulativa, que permanezca en hermosos razonamientos, pero ausente de una práctica concreta. Son ellos quienes en última instancia decidirán hacer o dejar de hacer algo, en plena libertad, y en ello radica su responsabilidad moral, de la cual no pueden escapar. Hoy día, la aldea global sin ética no logrará sobrevivir.

En este siglo XXI, Enfermería se ha insertado en este mundo globalizado con grandes desafíos, ya que la práctica se está volviendo cada vez más sofisticada y polivalente, dentro de un contexto multidisciplinario y con acciones muy flexibles. Las condiciones de la práctica profesional tienden a transformarse diariamente por los efectos del desarrollo tecnológico, de los sistemas de comunicación computarizados, por los cambios epidemiológicos del fenómeno del envejecimiento de la población y la aparición de nuevas en- fermedades como el SIDA y de otras que se creían ya erradicadas y que tienen estrecha relación con la pobreza.

Para servir en esta sociedad, hay que poner especial cuidado en la formación de la enfermera, ya que deben ser excelentes cuidadoras, y tener una gran sensibilidad para tratar a individuos en los estados de salud y enfermedad en diversos contextos socioculturales de la aldea global, pero paralelo a ello, deben de tener además capacidad de síntesis y de análisis y, desarrollar el pensamiento crítico junto a las competencias necesarias para las comunicaciones interpersonales y de sistemas computacionales.

Algunas autoras $(18,19)$ hacen especial énfasis en la formación de enfermeras en áreas tales como: la atención a la tercera edad, los cuidados entregados en el hogar, el área de la genética, la atención frente a los desastres naturales, los conflictos y las guerras, y la investigación internacional, destacándose el fenómeno de la violencia en el mundo y el de las drogas. Especial énfasis hay que poner en la calidad de los cuidados que se otorgan, teniendo siempre presente el paradigma del humanismo junto a los criterios éticos que deben dirigir sus comportamientos (20).

Este modelo de actuación de Enfermería requiere por lo tanto de preparación y talento científico, técnico y humano para adquirir estas cualidades. Hay que crear y construir permanentemente la profesión y tener imaginación para adaptar a la organización un entorno favorecedor del cuidado de las personas, además de sensibilidad y fortaleza para mantenerse frente al dolor de otros (21), así como cuidarlo en su sufrimiento (22).

Como personas y enfermeras en este mundo globalizado somos parte activa de ello, por lo que tenemos el desafío de engrandecer esta sociedad, pero no sólo respecto a la eficacia y el rendimiento, sino que a la capacidad de vivir lo bello (16) sin fronteras ni barreras, con solidaridad, en un camino de sanos y grandes encuentros. 


\section{CONCLUSIÓN}

En Enfermería se han producido cambios trascendentales a lo largo del siglo pasado que han culminado en un enfoque de cuidados de calidad y atención integral acogido por el paradigma humanista. La innovación en el ámbito de las responsabilidades y la orientación hacia la profesionalización han modelado el rol de la enfermera.

Para servir a la sociedad de hoy y la del futuro, en este mundo globalizado, es necesario planificar muy bien la formación de la enfermera con las diversas competencias que se requieren, con énfasis en los valores de responsabilidad, solidaridad y humanización, lo que significa otorgar dignidad al hombre y sentido a nuestra sociedad.

\section{REFERENCIAS}

1. Donahue PM. Historia de la Enfermería. Barcelona: España: Edit. Harcourt. 1988.

2. García C, Caro M, Martínez ML. Historia de la Enfermería. Madrid: Edit. Harcourt. 2001.

3. Amartya Sen. cit. In: Muñoz J. Chile y los desafíos presentes en sus nuevos escenarios económicos. Rev Universidad y Sociedad. Dic. 2002;4(24): 3-4.

4. Fielbaum G. Reflexiones sobre la Globalización y su impacto en Chile. Rev Universidad y Sociedad. Dic. 2002;4(24): 3438.

5. Robinson K. Una introducción a los derechos humanos de las personas migrantes. Madrid: Edit. Amnistía Internacional. España. 2006.

6. Albert M, Boissonnat J, Camdessus M. Nuestra fe en este siglo. Santiago de Chile: Ed. Desafío. 2004.

7. Vargas M. Tomar las riendas de la globalización. In: Albert M, Boissonnat J, Camdessus M. Nuestra fe en este siglo.
Santiago de Chile: Ed. Desafío. 2004.

8. Rodríguez C, Vio CP. Necesidad de Posgraduados en Chile. In: Consejo Superior de Educación. Estudios de Posgrado. Perspectivas y desafíos. Santiago. Chile. 2003; p.21-39.

9. Muñoz J. Chile y los desafíos presentes en sus nuevos escenarios económicos. Rev. Universidad y Sociedad. Dic. 2002; 4 (24):3-4.

10. Fromm Erich. El arte de Amar. Buenos Aires: Edit. Paidos. 1966.

11. Arroyo de Cordero G. Humanismo en Enfermería. Rev Enf. IMSS. 2000; 8(2).6163.

12. Quintero MC. Espiritualidad y Afecto. In:Pinto N. Cuidado y Práctica de Enfermería. España: Edit. Universidad Nacional de Colombia. 2000; p.184-191.

13. Abaunza de González M. Gerencia en el cuidado productivo de Enfermería. In: Pinto N. Cuidado y Práctica de Enfermería. España: Edit. Universidad Nacional de Colombia. 2000; p.49-56.

14. Colliere MF. Invisible Care and Invisible Women as Health Care-Providers. International Journal of Nursing Studies, 1986; 23(2):95-112.

15. Zárate R. La Gestión del Cuidado de Enfermería. Index de Enfermería. 2004;13 (44-45):42-46.

16. De la Rosa MA. ¿Qué es el postmodernismo? Rev.Lindaraja. Hallado en: http:// www.filosofiayliteratura.org/zen [Acceso julio 25 2007].

17. Fraile CG. Ciencia, Etica y Enfermería. Rev Ciencia y Enfermería. 2002; 8 (1):9-14.

18. Tschudin V. La voz futura de la enfermería. Rev Latino-Am Enfermagem. 2003;11 (4): 413-419.

19. Miotto MdaG. La contribución de la Enfermería frente al fenómeno de las drogas y la violencia en América Latina: un proceso de construcción. Rev Ciencia y Enfermería. 2002; 8(2):9-19.

20. Fernández J, Dumét R, Oliveira D, Vierira $\mathrm{T}$, Teixeira et al. Dimensão Etica de 
fazer cotidiano no processo de formação do enfermeiro. Rev Esc. Enferm USP. 2008;42.(2):396-403.

21. Urbina O, Pérez A, Delgado JE, Martín $X$. El modelo de actuación de Enfermería y su valor humanista. Educ Med Super. 2003;17(3).

22. Martín G. ¿Pero de lo que estamos hablando cuando abordamos la integridad? Rev Bras Enferm. Brasilia. 2008; 61(3):285. 